\title{
Closing-Wedge Distal Femoral Osteotomy Combined with Medial Patellofemoral Ligament Reconstruction for Recurrent Patellar Dislocation with Genu Valgum
}

\section{Lizhong Jing}

Department of Orthopedics $₫$ Affiliated Hospital to Shandong University of Traditional Chinese Medicine

\section{Xiaole Wang}

Department of Orthopedics $₫$ Affiliated Hospital to Shandong University of Traditional Chinese Medicine Kun Liu

Department of Orthopedics $\bowtie$ Penglai Traditional Chinese Medicine Hospital

\section{Xiaotan Wang}

Shandong University of Traditional Chinese Medicine

\section{Lu Jiang}

Shandong University of Traditional Chinese Medicine

\section{Di Wu}

Shandong University of Traditional Chinese Medicine

\section{Zhiwei Zhang}

Shandong University of Traditional Chinese Medicine

\section{Zhuang Li}

Shandong University of Traditional Chinese Medicine

\section{Le Yu}

Department of Orthopedics $₫$ Affiliated Hospital to Shandong University of Traditional Chinese Medicine

\section{Shaoshan Wang}

Department of Orthopedics $₫$ Affiliated Hospital to Shandong University of Traditional Chinese Medicine Jiushan Yang ( $\square$ yangjiushan001@126.com )

Department of Orthopedics $₫$ Affiliated Hospital to Shandong University of Traditional Chinese Medicine

\section{Research article}

Keywords: closing-wedge distal femoral osteotomy, medial patellofemoral ligament reconstruction, recurrent patellar dislocation, genu valgum

Posted Date: July 24th, 2020 
DOI: https://doi.org/10.21203/rs.3.rs-38833/v1

License: (c) (1) This work is licensed under a Creative Commons Attribution 4.0 International License. Read Full License

Version of Record: A version of this preprint was published at BMC Musculoskeletal Disorders on August 9th, 2021. See the published version at https://doi.org/10.1186/s12891-021-04554-5. 


\section{Abstract}

Background: Medial patellofemoral ligament reconstruction (MPFLR) is a well-established procedure for addressing recurrent patellar dislocation (RPD) in young patients. However, for RPD with genu valgum, simultaneous MPFLR and closing-wedge distal femoral osteotomy (CWDFO) may be a promising procedure yet rarely reported. This study's purpose was to observe and analyse the clinical and imaging findings of CWDFO combined with MPFLR for RPD with genu valgum.

Methods: From May 2015 to Apirl 2018, 25 patients with RPD and genu valgum were surgically treated in our department. Anteroposterior long-leg, weight-bearing, lower-extremity radiographs, lateral radiographs and computed tomography (CT) scans of the patellofemoral joint were obtained, and the femorotibial angle (FTA), mechanical lateral distal femoral angle (mLDFA), weight-bearing line rate (WBLR), patellar height, patellar lateral shift (PLS) and tibial tubercle-trochlear groove (TT-TG) distance were analysed. Validated knee scores, such as the Kujala, Lysholm, visual analogue scale (VAS) scores and Tegner socres, were evaluated preoperatively and 2 years postoperatively.

Results: 25 patients, with an average age of 19.8 years (14-27), were evaluated. All patients had been able to achieve a better sports level without any problems during the 2-year follow-up period. There has been no recurrence of patellar instability. Compared with preoperation, the FTA, mLDFA, WBLR and PLS showed statistically significant improvement following the procedure $(p<0.001)$. Meanwhile, no significant differences in the Insall index and TT-TG distance was found. The mean Kujala score, average Lysholm score, VAS score and Tegner socres showed significant improvement postoperatively.

Conclusions: CWDFO combined with MPFLR is a suitable treatment for RPD with genu valgum, as it leads to significant improvement in the clinical and imaging findings of the knee in the short term.

\section{Background}

RPD is very common in the among teenagers [1]. Numerous factors contribute to RPD, such as patella alta, femoral malrotation, tibial tubercle lateralization, and genu valgum[2, 3] Therefore, an individual treatment option is needed on the basis of the potential pathoanatomy.

Genu valgum has been proven to considerably influence patellar tracking[4]. For these patients, it may not be appropriate to apply medialization of the tibial tuberosity (TTM), which noticeably increased the patellofemoral and the medial tibiofemoral contact pressure, changed the balance of tibiofemoral joint loading[5]. MPFLR, is known as the most common surgical procedure for PRD, but genu valgum is one of further risk factors for failure of an isolated MPFLR[6, 7]. Besides, more than 2 times greater risk of degeneration in the lateral femorotibial compartment was found in the circumstances of malalignment of 3 degrees valgus $[8,9]$. Therefore, it may be reasonable to correct the valgus deformity simultaneously if this kind deformity is one of high risks for RPD. 
Generally speaking, both patellar instability and maltracking need to be corrected for RPD [8, 9]. In terms of patellar instability, the popularly accepted opinion is the MPFLR [10]. However, patellar maltracking could be restored by DFO in the patients with genu valgum[11]. So, CWDFO combined MPFLR could be justified in these patients with genu valgum.

However, recent studies examining the clinical and imaging findings of the treatment of RPD due to valgus deformity by DFO combined with MPFLR are rare. And there are no studies with a larger number of cases available. This study's purpose was to analyse the short-term results of CWDFO combined with MPFLR for RPD with genu valgum. We assumed that the satisfactory clinical and imaging results can be obtained by this procedure in these patients after a minimum follow-up period of 2 years. As far as we know, this study has the largest cohort of these disorders any study ever published.

\section{Methods}

\subsection{Study design}

The indication for surgical treatment was RPD ( $\geq 2$ dislocations) of the knee combined with genu valgum deformity. The exclusion criteria were acute lateral patellar dislocation, habitual patellar dislocation, and combination with patella alta or femoral intorsion.

The study was approved by the Ethics Committee of our hospital. All patients signed an informed consent form.

There was no control group.

\subsection{Clinical and Radiological Evaluation}

All patients were evaluated preoperatively and 2 years postoperatively according to the Kujala, Lysholm, visual analogue scale (VAS) scores and Tegner scores. The preoperative and 2-year postoperative radiographic examinations of the knee included anteroposterior (AP) long-leg, weight-bearing, lowerextremity radiographs and lateral radiographs to assess the femorotibial angle (FTA), mechanical lateral distal femoral angle (mLDFA), weight-bearing line rate (WBLR) and patellar height. The patellar height was calculated by the method described by Insall [12]. The patellar lateral shift (PLS) preoperatively and 2 years postoperatively was measured by CT at 30 degrees of knee flexion, as described by Nha et al[11], and was defined as the shortest distance from the cortex of the lateral trochlea to the lateral edge of the patella. The demographic data of these patients are presented in Table 1.

\subsection{Surgical technique}

Initially, arthroscopy was performed, confirming patellar maltracking and remove any loose bodies.

For CWDFO, preoperative planning was performed by the method described by Paley et al[13]. Our target line passed $50 \%$ of the plateau width from the inside margin to that of outside. The operative 
manipulation was carried out following the method presented by Frings et al [10]. The TomoFix plate was used to stabilize the osteotomy area(Fig. 1a, b).

The MPFLR was performed in all patients according to Schoettle's technique[14]. The autologous semitendinosus graft was fixed in the medial margin of patella by two absorbable suture anchors and in the anatomical femoral insertion site by an unabsorbable interference screw (Fig. 1c, d).

Subsequently, patellar tracking was re-evaluated. Arthroscopic lateral release with a thermal device was then performed if a strong patellar tilt and lateral soft tissue tightness were still observed.

\subsection{Rehabilitation}

A standardized rehabilitation protocol was performed. Second day postoperation, passive knee flexion, the quadriceps contraction isometrically and ankle pumps were carried out according to the perscription. Four weeks after surgery, patients were allowed to start partial weight-bearing exercise. Progressive passive flexion was built up for the first 6 weeks. The knee brace was worn for 6 weeks after surgery. After 8 weeks, all patients were instructed to a gradual return to full weight-bearing. After 4 months, noncompetitive sports was allowed.

\subsection{Statistical analysis}

The preoperative and postoperative results for all cases were compared using paired tests. Variables compared on the basis of independent sample tests included the Lysholm scores, Kujala scores, VAS scores, Tegner scores, FTA, mLDFA, WBLR, Insall index, PLS and TT-TG. The average data of these measurements were used in the analysis process,. In this study, SPSS (version 24, SPSS, Inc., Chicago, Illinois) was used to perform the statistical analysis. All reported $P$ values are 2 -tailed, and $P$ values less than 0.05 were considered to indicate statistical significance.

Figure 1 Intraoperative setting of the CWDFO. Removing the wedge (a). Closing the medial osteotomy carefully (b). Performing osteosynthesis with a plate, and fixing a semitendinosus graft in the patella and anatomical femoral insertion site $(c, d)$.

Figure 2A 19-year-old girl with RPD and valgus deformity of the lower limb. The alignment of the leg axis, TT-TG distance, PLS and insall index of preoperation $(a, c, e, g)$ and postoperation $(b, d, f, h)$, respectively.

Figure 3A 16-year-old boy with RPD and valgus deformity of the lower limb. The alignment of the leg axis, TT-TG distance, PLS and insall index of preoperation $(a, c, e, g)$ and postoperation $(b, d, f, h)$, respectively. 


\section{CWDFO, MPFLR CWDFO, MPFLR, LR}

Total knees 1411

Age (years) 18 (14-27) 20 (15-26)

Male 67

Female 84

Preoperative FTA $\left({ }^{\circ}\right) 163.43 \pm 3.84166 .01 \pm 4.59$

Correction angle $10.17 \pm 2.138 .75 \pm 1.71$

Postoperative FTA $\left(^{\circ}\right) 174.10 \pm 3.04176 .38 \pm 2.55$

TT-TG $(\mathrm{mm}) 16.33 \pm 2.1619 .07 \pm 3.05$

TT medialization $(\mathrm{mm})---$

Insall index $1.03 \pm 0.241 .09 \pm 0.16$

Follow-up duration (months) 35(24-57) 37(27-51)

CWDFO, closing-wedge distal femoral osteotomy; LR, lateral release; FTA, femorotibial angle; MPFLR, medial patellofemoral ligament reconstruction; TT-TG, tibial tubercle-trochlear groove; TT, tibial tuberosity

\section{Results}

From May 2015 to Apirl 2018, 27 patients underwent CWDFO combined with MPFLR at our institution. Two patients were unwilling to take further part in the clinical follow-up period after 1 year postoperatively. 25 of 27 patients (92.5\%, 9 women, 16 men) were available for more than 2 years of clinical follow-up observation, with an average age of 19.8 years (14-27) and an average follow-up duration of 36.67 months (24-57). 15 patients $(60 \%)$ had right-sided patellar instability, while ten ( $40 \%)$ had instability on the left side. All patients returned to non-competitive sporting activities without any problems. There has been no RPD. 
Table 2

Preoperative and postoperative radiological parameters

\section{Scale Preoperatively 2 years postoperatively $\mathrm{p}$ value}

FTA $\left(^{\circ}\right) 164.67 \pm 3.04174 .53 \pm 2.42<0.001$

WBLR (\%) $74.13 \pm 3.6850 .20 \pm 2.76<0.001$

$\operatorname{mLDFA}\left({ }^{\circ}\right) 81.13 \pm 1.6886 .93 \pm 1.98<0.001$

Insall $1.04 \pm 0.121 .03 \pm 0.110 .712$

PLS (mm) $12.53 \pm 2.424 .60 \pm 1.50<0.001$

TT-TG $(\mathrm{mm}) 17.20 \pm 3.8417 .53 \pm 3.200 .739$

WBLR, weight-bearing line rate; mLDFA, mechanical lateral distal femoral angle; PLS, patellar lateral shift

Table 3

Preoperative and postoperative knee scores for patients

Scale Preoperatively 2 years postoperatively $p$ value

Lysholm $47.87 \pm 5.2584 .27 \pm 4.11 \rrbracket 0.001$

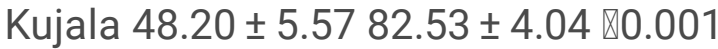

VAS $5.07 \pm 1.281 .80 \pm 1.2 \otimes 0.001$

Tegner $2(1-4) 4(3-6) \otimes 0.001$

VAS, visual analogue scale

Compared with preoperation, following the procedure, the FTA, WBLR, MLDFA and PLS showed statistically significant improvement $(p<0.001)$. Meanwhile, no significant differences in the Insall index and TT-TG distance was found at 2 years postoperatively. The results are specified in Table 2, Fig. 2, and Fig. 3.

The mean Kujala score, average Lysholm score, VAS score and Tegner score are presented in Table 3. All applied knee scores showed statistically significant improvement postoperatively.

We found complications in only one patient, who developed knee stiffness 12 weeks postoperatively and achieved full range of motion after manipulation under anaesthesia. None of the patients developed infection or non-union from the surgical procedure.

\section{Discussion}

The most important finding of this study is that the treatment of RPD with genu valgum by CWDFO combined with MPFLR is effective.The procedure can obtain radiological correction of the patellofemoral 
instability and genu valgum, improvement in clinical scores.

RPD is connected to various anatomic abnormalities, for instance, trochlear dysplasia, femoral malrotation, tibial tubercle lateralization, and genu valgum. Because of the countless risk factors for patellar instability, a multitude of techniques have been introduced to solve this problem; the optimal procedure, still, has caused a heated debate .

It is now well established that TTM and isolated MPFLR are effective procedures to treat RPD[15-17], but these approaches are also imperfect. In terms of MPFLR, Shah et al[18] revealed an overall cumulative complication rate of $26.1 \%$ when this operation is adopted. Common complications included fracture of patellar, residual instability, pain and flexion loss. Up to $38-40 \%$ of cases were reported having anterior knee pain in those underwent MPFLR[19]. In additional, Gobbi et al[20] suggested patellar shift and tilt correction will not improve significantly when performing isolated MPFLR in patients with RPD, while patellar maltracking is regard as a pathoanatomic risk factor for RPD[21, 22].

Several authors stated that because of altered contact pressures, TTM is followed by osteoarthritis of the patellofemoral and tibiofemoral joints. Kuroda et al[5] reported in the cadaveric study that TTM significantly increased the contact pressure of patellofemoral compartment and the medial tibiofemoral compartment. Lobner et al[23] found patients treated by TTM revealed an increased damage of retropatellar cartilage and worsen pain during activity. Mani et al[24] considered the pressure applied to tibiofemoral cartilage could be altered by tibiofemoral kinematic changes after TTM in their controlled laboratory study.

Axis deviations of the lower extremity such as genu valgum is one of further risk factors for failure of an isolated MPFLR[6, 7]. Because genu valgum has been regarded as one of negative factors to increase the forced shift of the patella towards the lateral side in terminal knee extension and result in a J-sign[9, 25]. The MPFL acts as a checkrein to lateral translation of the patella yet it does not pull the patella into the trochlear groove, hence isolated MPFLR should be avoided in the patients of genu valgum[26].

Those problems mentioned above could be resolved by DFO in the patients with associated genu valgum deformity.Traditionally, DFO was regarded as a surgical option for lateral tibiofemoral compartment osteoarthritis[27]. However, in recent years, it has been commonly accepted that correction of genu valgum could reduce the lateral vector applied to the patella, thereby gaining stabilization of the patella and decreasing the risk of RPD[11]. It is of great importance because genu valgum is an accepted risk factor for RPD, and also very common among teenagers. Nha et al[11] investigated the outcomes of 14 patients who underwent CWDFO over a mean follow-up period of 30 months. The mLDFA changed significantly from 83 to 89 . The average distance of the PLS declining from $13.5 \mathrm{~mm}$ to $2.0 \mathrm{~mm}$. All patients' subjective symptoms were significantly improved after the operation, and there was no RPD. Dickschas[28] studied 18 patients who underwent CWDFO over an average follow-up period of 44 months, and no redislocation was found after surgery. The VAS score for anterior knee pain improved from 5.6 to 2.1 . 
DFO alone or in combination with different soft tissue procedures for RPD has been reported. The treatment of RPD with CWDFO was first reported by Omidi et al[29] in 2009. The author found that the correction of genu valgum deformity alone can improve the patellofemoral congruence angle and reduce the probability of patellar dislocation. Since then, many studies on this procedure have been reported. Chang et al[27] examined 10 patients who underwent CWDFO with lateral retinacular release and tightening of the medial retinaculum over an average follow-up period of 20 months. The postoperative KSS and Kujala scores were significantly improved. Patellar dislocation did not recur. Purushothaman et al[30] reported the case of an RPD patient treated by OWDFO combined with MPFLR who achieved satisfactory postoperative results at the 1-year follow-up examination. Frings $\mathrm{J}$ [22] reported the results of 12 patients with RPD of genu valgus treated with CWDFO combined with MPFLR and TTM. The VAS score, Kujala score, Lysholm score and Tegner score were significantly improved compared with those before the operation, and there was no RPD. We believe that DFO alone only converts complex patellar dislocation to simple patellar dislocation in RPD patients with genu valgum deformity. Without associated soft tissue repair, MPFLR failure and femoral trochlear abnormalities are potential risks for RPD. Furthermore, MPFLR results in higher functional scores than medial retinacular constriction[31]. Therefore, we chose to perform CWDFO combined with MPFLR in such patients, as previously described, and our results verify the reliability of this procedure.

McWalter et al[4] stated that varus or valgus deformities of the leg axis could affect patellar tracking remarkably. Specifically, it has a strong impact on the patellar tilt, yet no significant difference was found in the level or slope of lateral translation[4]. However, Nha et al [11] found that CWDFO could decreased the mean distance of PLS reduced from $13.5 \mathrm{~mm}$ to $2.0 \mathrm{~mm}$. Similarly, Our finding is consistent with Nha's conclusions in this respect. Since MPFLR does not improve patellar tilt or shift[20],the rationale for DFO maybe the reduction of the $Q$ angle and medialization of the patellar tendon insertion [32].

Some authors stated that a TT-TG distance $>15 \mathrm{~mm}$ is a risk factor for RPD[33] and a realignment procedure is recommended to be performed if TT-TG distance $>20 \mathrm{~mm}$ [34]. However, to date, there is still no clear evidence of whether TTM is necessary when performing MPFLR in patients with RPD. According to the findings of Ostermeier et al[35], TTM showed no significant relief of ligament loading and stabilizing effect on patellar movement. Matsushita et al[36] demonstrated that it yielded similar clinical results regardless of TT-TG distance greater than $20 \mathrm{~mm}$ or not if isolated MPFLR performed in RPD. They deduced that a TT-TG distance $>20 \mathrm{~mm}$ may not be an absolute operative indication for TTM. Interestingly, none of patients performing TTM, but Nha et al[11] found that DFO could affect the TT-TG distance, which declined from $20.4 \mathrm{~mm}$ preoperatively to $13.5 \mathrm{~mm}$. None of our patients underwent TTM in our study, however, in contrast to the results reported by Nha et al, we found no significant change in the TT-TG distance between before and after surgery. We attributed this finding to the level of the osteotomy area in our study. Our osteotomies were all proximal to the femoral trochlea and therefore did not affect the relative relationship of the femoral trochlea and the tibial tubercle.

This study also has limitations. First, this study was retrospective and did not have a control group performing other surgical techniques such as isolated MPFLR, TTM, just to name a few, but as Nha[11] 
mentioned, CWDFO may be regarded as one of the surgical options for RPD with genu valgum since there were the remarkable improvement in clinical outcomes and radiological results. Second, the present study had a relatively small sample size and short follow-up period, However, a 2-year follow-up period might be enough to prove an acceptable clinical outcomes. Further investigations with a larger sample size and longer follow-up period are needed.

\section{Conclusion}

Great clinical and radiological results can be obtained in the treatment of RPD with genu valgum by CWDFO combined with MPFLR. During the 2-year follow-up period, all patients in our study maintained the stability of the patellofemoral compartment, and none of patients suffered redislocation. The clinical scores and radiological evaluations, except for the TT-TG distance and Insall index, showed significant improvement postoperatively.

\section{Abbreviations}

MPFLR, Medial patellofemoral ligament reconstruction; RPD, recurrent patellar dislocation; CWDFO, closing-wedge distal femoral osteotomy; TTM, medialization of the tibial tuberosity; FTA, femorotibial angle; mLDFA, mechanical lateral distal femoral angle; WBLR, weight-bearing line rate; PLS, patellar lateral shift; TT-TG, tibial tubercle-trochlear groove; VAS, visual analogue scale;

\section{Declarations}

\section{Ethics approval and consent to participate}

This study was approved by the Institutional Ethics committee of Affiliated Hospital to Shandong University of TCM, China (No. 2019-025-KY). A certificate of approval has been provided. The requirement of informed consent was exempted due to the retrospective nature of the study.

\section{Consent for publication}

Not applicable.

\section{Availability of data and materials}

The datasets analyzed in this study are available from the corresponding author on reasonable request.

\section{Competing interests}

The authors declare that they have no competing interests.

\section{Funding}

None.

\section{Authors' contributions}

LZJ and JSY were responsible for the idea and design of the study. SSW planned the Study protocol. XLW, KL performed all operations mentioned in the study. XTW, LJ, DW were responsible for data 
acquisition. ZWZ, ZL, LY did the statistical analysis and interpreted the results. LZJ wrote the Manuscript. All authors read and approved the final manuscript.

\section{Acknowledgments}

None.

\section{References}

1. Tan S, Tan L, Lim A, Hui JH. Hemiepiphysiodesis is a potentially effective surgical management for skeletally immature patients with patellofemoral instability associated with isolated genu valgum. Knee Surg Sports Traumatol Arthrosc. 2019;27:845-9.

2. Kita K, Tanaka Y, Toritsuka Y, Amano H, Uchida R, Shiozaki Y, et al. 3D Computed Tomography Evaluation of Morphological Changes in the Femoral Tunnel After Medial Patellofemoral Ligament Reconstruction With Hamstring Tendon Graft for Recurrent Patellar Dislocation. Am J Sports Med. 2017;45:1599-607.

3. Malecki K, Fabis J, Flont P, Lipczyk Z, Niedzielski K. Preliminary results of two surgical techniques in the treatment of recurrent patellar dislocation: Medial patellofemoral ligament reconstruction versus combined technique of vastus medialis advancement, capsular plasty and Roux-Goldthwait procedure in treatment of recurrent patellar dislocation. INT ORTHOP. 2016;40:1869-74.

4. McWalter EJ, Cibere J, Maclntyre NJ, Nicolaou S, Schulzer M, Wilson DR. Relationship between varusvalgus alignment and patellar kinematics in individuals with knee osteoarthritis. J BONE JOINT SURG AM. 2007;89:2723-31.

5. Kuroda R, Kambic H, Valdevit A, Andrish JT. Articular cartilage contact pressure after tibial tuberosity transfer. A cadaveric study. Am J Sports Med. 2001;29:403-9.

6. Nelitz M, Theile M, Dornacher D, Wolfle J, Reichel H, Lippacher S. Analysis of failed surgery for patellar instability in children with open growth plates. Knee Surg Sports Traumatol Arthrosc. 2012;20:822-8.

7. Gesslein M, Roessler PP, Schuttler KF, Biber R, Bail HJ, Efe T. Complications and failure of MPFL reconstruction with free tendon grafts in cases of patellofemoral instability. TECHNOL HEALTH CARE. 2015;23:659-66.

8. Felson DT, Niu J, Gross KD, Englund M, Sharma L, Cooke TD, et al. Valgus malalignment is a risk factor for lateral knee osteoarthritis incidence and progression: findings from the Multicenter Osteoarthritis Study and the Osteoarthritis Initiative. Arthritis Rheum. 2013;65:355-62.

9. Frosch $\mathrm{KH}$, Schmeling A. A new classification system of patellar instability and patellar maltracking. Arch Orthop Trauma Surg. 2016;136:485-97.

10. Frings J, Krause M, Akoto R, Wohlmuth P, Frosch KH. Combined distal femoral osteotomy (DFO) in genu valgum leads to reliable patellar stabilization and an improvement in knee function. Knee Surg Sports Traumatol Arthrosc. 2018;26:3572-81. 
11. Nha KW, Ha Y, Oh S, Nikumbha VP, Kwon SK, Shin WJ, et al. Surgical Treatment With Closing-Wedge Distal Femoral Osteotomy for Recurrent Patellar Dislocation With Genu Valgum. Am J Sports Med. 2018;46:1632-40.

12. Insall J, Salvati E. Patella position in the normal knee joint. RADIOLOGY. 1971;101:101-4.

13. Paley D, Pfeil J. [Principles of deformity correction around the knee]. ORTHOPADE. 2000;29:18-38.

14. Schottle PB, Schmeling A, Rosenstiel N, Weiler A. Radiographic landmarks for femoral tunnel placement in medial patellofemoral ligament reconstruction. Am J Sports Med. 2007;35:801-4.

15. Neri T, Parker DA, Beach A, Gensac C, Boyer B, Farizon F, et al. Medial patellofemoral ligament reconstruction with or without tibial tubercle transfer is an effective treatment for patellofemoral instability. Knee Surg Sports Traumatol Arthrosc. 2019;27:805-13.

16. Schneider DK, Grawe B, Magnussen RA, Ceasar A, Parikh SN, Wall EJ, et al. Outcomes After Isolated Medial Patellofemoral Ligament Reconstruction for the Treatment of Recurrent Lateral Patellar Dislocations: A Systematic Review and Meta-analysis. Am J Sports Med. 2016;44:2993-3005.

17. Tensho K, Shimodaira H, Akaoka Y, Koyama S, Hatanaka D, Ikegami S, et al. Lateralization of the Tibial Tubercle in Recurrent Patellar Dislocation: Verification Using Multiple Methods to Evaluate the Tibial Tubercle. J BONE JOINT SURG AM. 2018;100:e58.

18. Shah JN, Howard JS, Flanigan DC, Brophy RH, Carey JL, Lattermann C. A systematic review of complications and failures associated with medial patellofemoral ligament reconstruction for recurrent patellar dislocation. Am J Sports Med. 2012;40:1916-23.

19. Feller JA, Richmond AK, Wasiak J. Medial patellofemoral ligament reconstruction as an isolated or combined procedure for recurrent patellar instability. Knee Surg Sports Traumatol Arthrosc. 2014;22:2470-6.

20. Gobbi RG, Demange MK, de Avila L, Araujo FJ, Moreno RA, Gutierrez MA, et al. Patellar tracking after isolated medial patellofemoral ligament reconstruction: dynamic evaluation using computed tomography. Knee Surg Sports Traumatol Arthrosc. 2017;25:3197-205.

21. Rezvanifar SC, Flesher BL, Jones KC, Elias JJ. Lateral patellar maltracking due to trochlear dysplasia: A computational study. KNEE. 2019;26:1234-42.

22. Frings $\mathrm{J}$, Krause M, Akoto R, Frosch KH. Clinical Results after Combined Distal Femoral Osteotomy in Patients with Patellar Maltracking and Recurrent Dislocations. J KNEE SURG. 2019;32:924-33.

23. Lobner S, Krauss C, Reichwein F, Patzer T, Nebelung W, Venjakob AJ. Surgical treatment of patellar instability: clinical and radiological outcome after medial patellofemoral ligament reconstruction and tibial tuberosity medialisation. Arch Orthop Trauma Surg. 2017;137:1087-95.

24. Mani S, Kirkpatrick MS, Saranathan A, Smith LG, Cosgarea AJ, Elias JJ. Tibial tuberosity osteotomy for patellofemoral realignment alters tibiofemoral kinematics. Am J Sports Med. 2011;39:1024-31.

25. Zhang Z, Zhang H, Song G, Zheng T, Feng H. A pre-operative grade $3 \mathrm{~J}$-sign adversely affects shortterm clinical outcome and is more likely to yield MPFL residual graft laxity in recurrent patellar dislocation. Knee Surg Sports Traumatol Arthrosc 2019. 
26. Caplan N, Nassar I, Anand B, Kader DF. Why Do Patellofemoral Stabilization Procedures Fail? Keys to Success. Sports Med Arthrosc Rev. 2017;25:e1-7.

27. Chang CB, Shetty GM, Lee JS, Kim YC, Kwon JH, Nha KW. A Combined Closing Wedge Distal Femoral Osteotomy and Medial Reefing Procedure for Recurrent Patellar Dislocation with Genu Valgum. YONSEI MED J. 2017;58:878-83.

28. Dickschas J, Ferner F, Lutter C, Gelse K, Harrer J, Strecker W. Patellofemoral dysbalance and genua valga: outcome after femoral varisation osteotomies. Arch Orthop Trauma Surg. 2018;138:19-25.

29. Omidi-Kashani F, Hasankhani IG, Mazlumi M, Ebrahimzadeh MH. Varus distal femoral osteotomy in young adults with valgus knee. J ORTHOP SURG RES. 2009;4:15.

30. Purushothaman B, Agarwal A, Dawson M. Posttraumatic chronic patellar dislocation treated by distal femoral osteotomy and medial patellofemoral ligament reconstruction. ORTHOPEDICS. 2012;35:e1668-72.

31. Zhao J, Huangfu X, He Y, Liu W. Recurrent patellar dislocation in adolescents: medial retinaculum plication versus vastus medialis plasty. Am J Sports Med. 2012;40:123-32.

32. Swarup I, Elattar O, Rozbruch SR. Patellar instability treated with distal femoral osteotomy. KNEE. 2017;24:608-14.

33. Grawe B, Stein BE. Tibial Tubercle Osteotomy: Indication and Techniques. J KNEE SURG. 2015;28:279-84.

34. Sherman SL, Erickson BJ, Cvetanovich GL, Chalmers PN, Farr JN, Bach BJ, et al. Tibial Tuberosity Osteotomy: Indications, Techniques, and Outcomes. Am J Sports Med. 2014;42:2006-17.

35. Ostermeier S, Stukenborg-Colsman C, Hurschler C, Wirth CJ. In vitro investigation of the effect of medial patellofemoral ligament reconstruction and medial tibial tuberosity transfer on lateral patellar stability. ARTHROSCOPY. 2006;22:308-19.

36. Matsushita T, Kuroda R, Oka S, Matsumoto T, Takayama K, Kurosaka M. Clinical outcomes of medial patellofemoral ligament reconstruction in patients with an increased tibial tuberosity-trochlear groove distance. Knee Surg Sports Traumatol Arthrosc. 2014;22:2438-44.

\section{Figures}



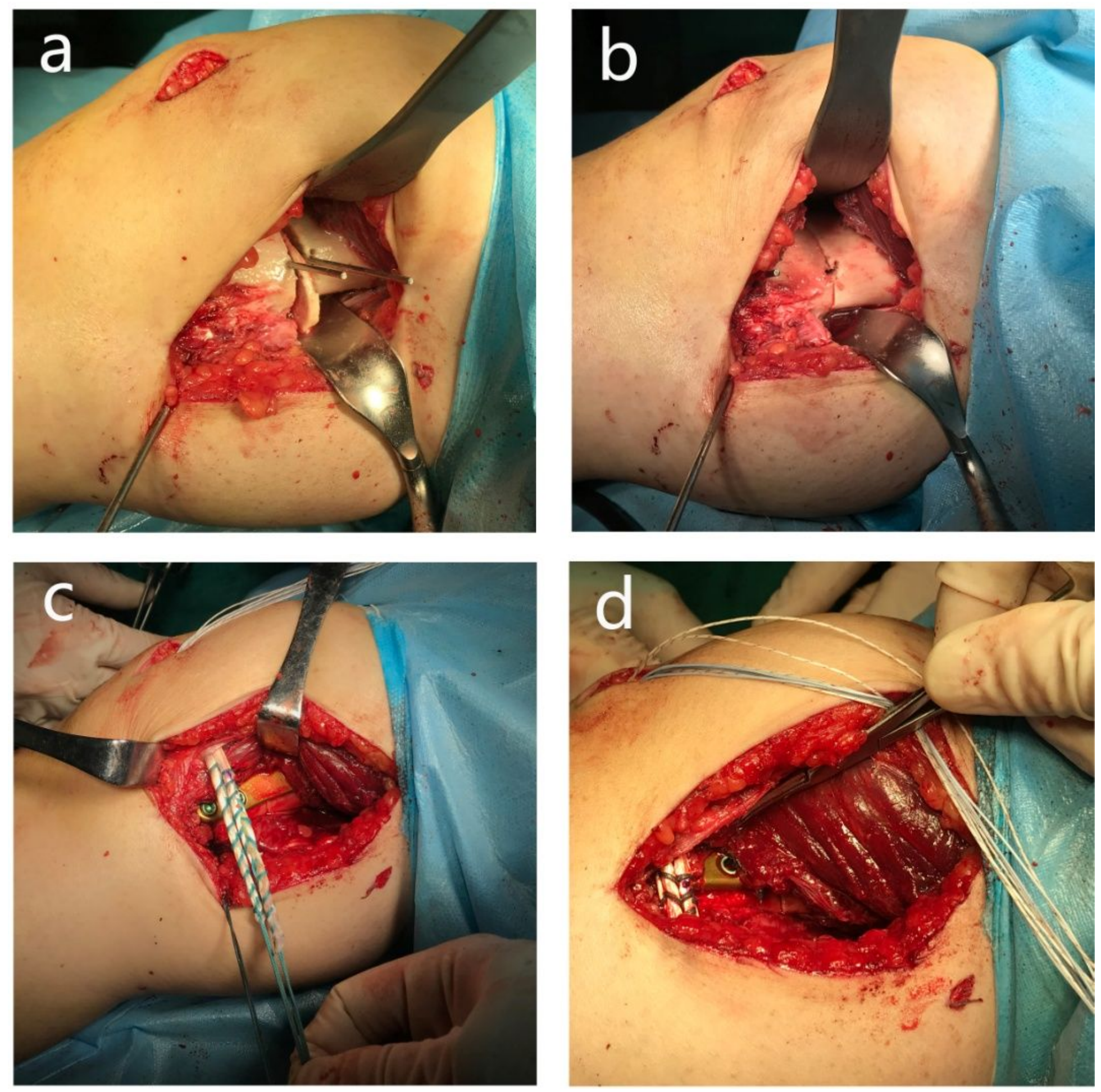

\section{Figure 1}

Intraoperative setting of the CWDFO. Removing the wedge (a). Closing the medial osteotomy carefully (b). Performing osteosynthesis with a plate, and fixing a semitendinosus graft in the patella and anatomical femoral insertion site $(c, d)$. 

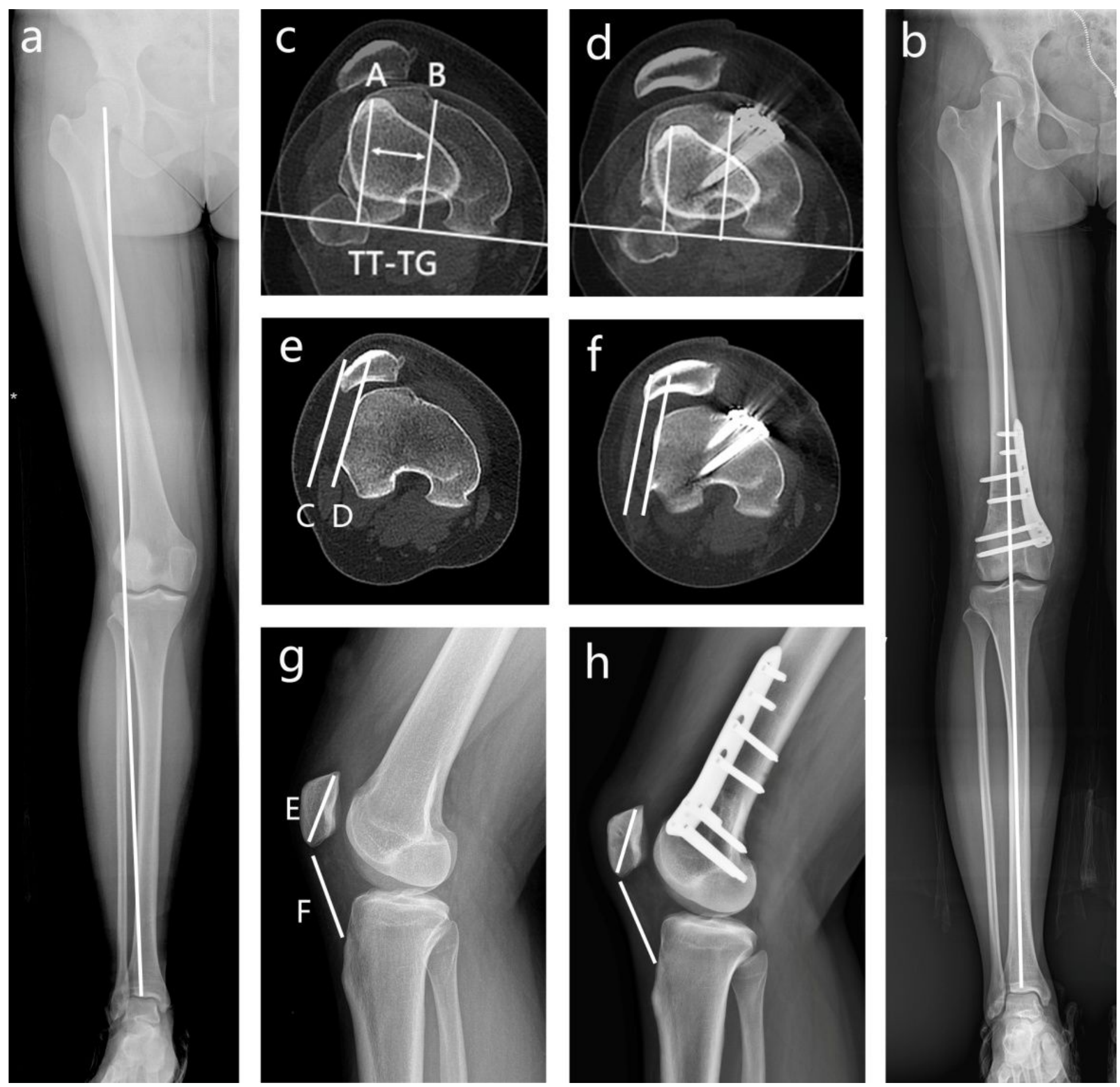

Figure 2

A 19-year-old girl with RPD and valgus deformity of the lower limb. The alignment of the leg axis, TT-TG distance, PLS and insall index of preoperation $(a, c, e, g)$ and postoperation $(b, d, f, h)$, respectively. 

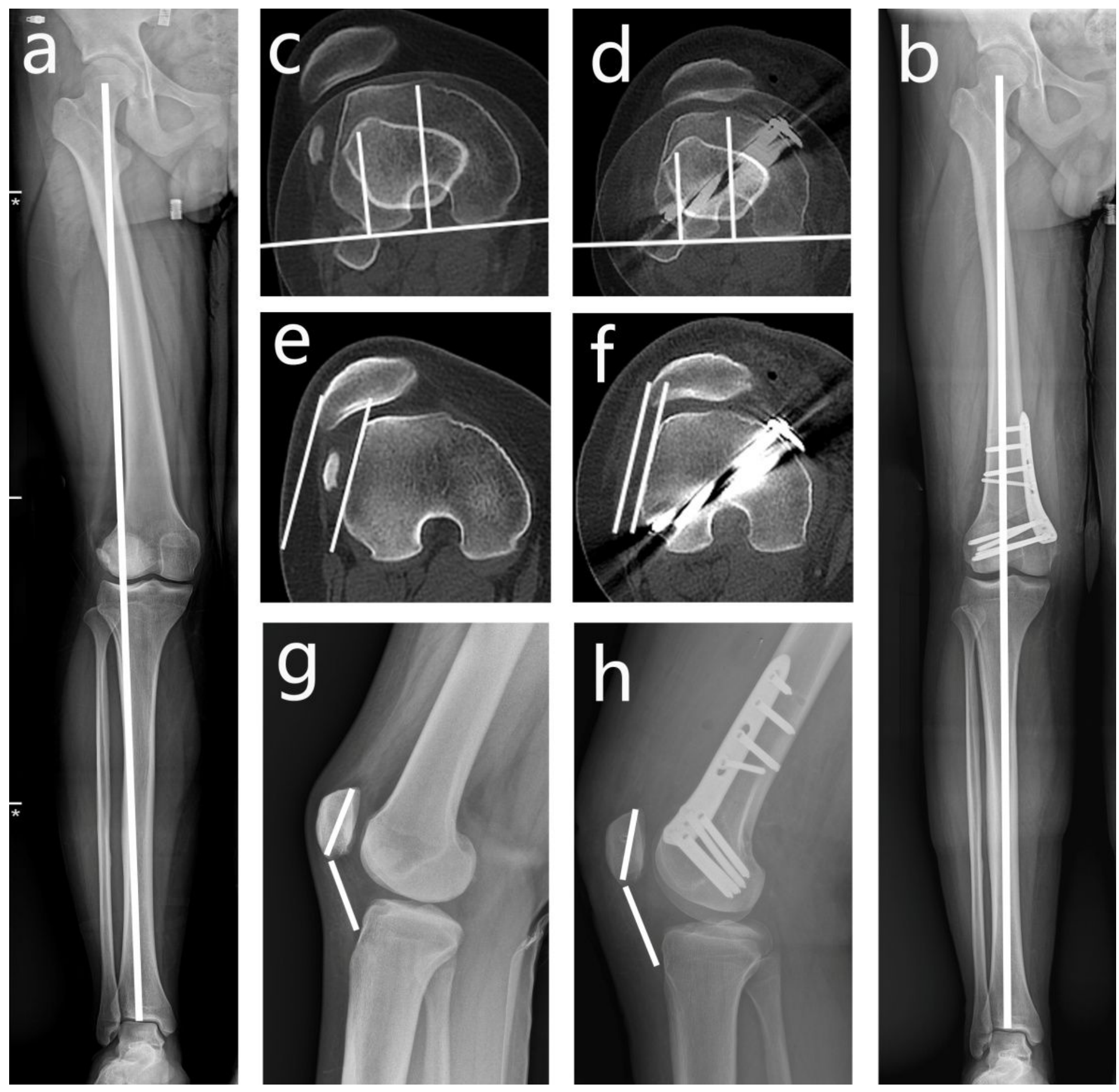

Figure 3

A 16-year-old boy with RPD and valgus deformity of the lower limb. The alignment of the leg axis, TT-TG distance, PLS and insall index of preoperation $(a, c, e, g)$ and postoperation $(b, d, f, h)$, respectively. 